\title{
Transoral approach for choanal atresia repair: a new surgical innovation in the making
}

\section{Background of invention}

Choanal atresia is a congenital obstruction of the back of the nose (posterior choanae), which prevents communication between the nose and nasopharynx. The incidence is around 1 in every 5000-7000 births. ${ }^{1}$ It results from failure of canalization of posterior choanae and persistence of nasobuccal and buccopharyngeal membranes. This posterior nasal obstruction in newborns if unilateral may pass unnoticed. Years later a child may present with unilateral nasal discharge or nasal obstruction. When the atresia is bilateral, it presents a life-threatening situation that has to be dealt with in the early days of life. ${ }^{2}$ That is because neonates are obligate nasal breathers. The diagnosis of bilateral choanal atresia usually is made immediately after birth, as cyanosis is evident. Crying interrupts the cyanosis. As the newborn cries, air passes into the lungs and oxygen saturation improves. Once it stops crying, cyanosis returns. This is known as cyclical cyanosis, which is characteristic for nasal obstruction in neonates mainly due to choanal atresia. Other causes maybe nasal pyriform aperture stenosis, mid-nasal stenosis, septal deviation, or nasal masses. Failure to pass nasal suction catheters past seven centimeters raises suspicion for choanal atresia. Mirror fogging and more recently naso-endoscopy confirms the diagnosis. A CT scan of the nasal cavity is vital in identifying the structural abnormality encountered. These abnormalities are the atretic plate, which may be bony or membranous and bony, thick vomer bone, impingement of medial ptyregoid plates, and narrow nasal cavity. The identification of specific structural abnormality may help in tailored surgery.

After stabilization of the patient either by endotracheal intubation or McGovern nipple to keep the mouth open, surgery is performed on an urgent basis in the first week of life. Traditionally, repair of choanal atresia was done initially blindly using puncture dilators through the nose. Emmert first described this technique in 1854. Urethral sounds or Fearson dilators were used to puncture the atretic plate. Recurrence rates were high and required multiple surgeries in order to achieve patency. In addition, complications from such a technique were dangerous. Injury to the skull base with potential cerebrospinal fluid leak or meningitis is drastic in neonates. Also false passage into soft palate may occur giving a false impression of successful puncture of atresia. In bony atresia, this technique is domed for failure.

Subsequently, repair was done via the transpalatal approach in the sixties. ${ }^{1,2}$ This approach involves incising the soft palate to reach the posterior choanae. Mucosal laps based on greater palatine vessels are raised to expose the posterior choanae. The exposure is wide and allows for drilling of atretic plate, vomer, and even the pterygoid plates. This approach was successful and showed lower recurrence rates but unfortunately had more complications. Complications of this approach include wound dehiscence, fistula formation, velopharyngeal insufficiency, and interruption of palatal growth. These interruptions lead to dentition problems and cross-bite deformity in children. Although a success rate from the first try was high reaching $84 \%$, complications were significantly higher than control groups. ${ }^{3}$ This approach is of historic interest and rarely performed nowadays.

With the advent of endoscopic techniques, surgeons used small
Volume 10 Issue 2 - 2018

\author{
Talal Al-Khatib \\ Department of Otolaryngology-Head Neck Surgery, King \\ Abdulaziz University, Saudi Arabia
}

Correspondence: Talal Al-Khatib, Department of Otolaryngology-Head Neck Surgery, Vice dean of Medicine, lbin Sina National College, Consultant Paediatric Otolaryngologist, Jeddah, Saudi Arabia, Email talkhatib@gmail.com ORCID 0000000287I05694

Received: October 30, 2017 | Published: April 17, 2018

endoscopes to reach the posterior choanae transnasally. This approach "Transnasal" became more popular and almost replaced the transpalatal approach. ${ }^{2,4}$ Recurrence of choanal atresia after transnasal approach is more common $(30-40 \%)$ than the old transpalatal approach but due to fewer complications, surgeons have stuck with the least invasive technique. ${ }^{4,5}$ Recurrence has been shown to be more common if the endoscopic procedure was done in the neonatal period (first 30 days of life). The reason for recurrence is thought to be due to the young age of neonates and possible intrinsic factors that led to the stenosis in the first place playing a role in restenosis.

I believe that the recurrence in the neonatal period is due to difficult access and incomplete removal of atretic plate (stenosis) rather than the young age of children itself, contrary to current believe. ${ }^{1,7}$ In addition, neonates have small nasal passages and transnasal approach might be difficult especially if endoscopic shaver or drilling of lateral pterygoid plates is required. Associated nasal abnormalities such as mid-nasal stenosis make transnasal approach even more difficult. Many modifications of the transnasal approach were used to improve the success rate including flap techniques and the use or disuse of nasal stents. ${ }^{4,8}$ Others suggested routine resection of the vomer as part of the procedure. ${ }^{7}$ These modifications of transnasal approach trying to improve the success rate imply that it is not the perfect approach.

\section{Invention idea}

Therefore, I present a new " transoral" approach that will allow sufficient access to posterior choanae with less trauma to the neonate's nasal cavities (Figure 1). Transoral view of the nasopharynx is not new. Surgeons used a 120 degree angled telescope to ensure correct placement of transnasal puncture during transnasal approach. Yet, repair of choanal atresia transorally is never been described. The idea proposed is to carry out the entire choanal atresia repair via the transoral route. This requires especially designed curved instruments to reach the choanal atresia but from the nasopharyngeal side instead of the nasal side.

\section{Description of procedure}

After endotracheal intubation, a dingman mouth gag retractor is inserted to retract the tongue. A 120 degree angled telescope is inserted behind the soft palate into the nasopharynx to visualize the posterior 
choanae. A curved drill is inserted transorally (Figure 1). Under direct vision, drilling of the atretic plate is carried out from the vomer medially (posterior nasal septum) till lateral pterygoid plate laterally. Care is taken not to drill deep into the nasal cavity to avoid injury to the posterior end of the inferior turbinate. This approach might be extremely helpful in thickened vomer bone where a reduction in size is essential.

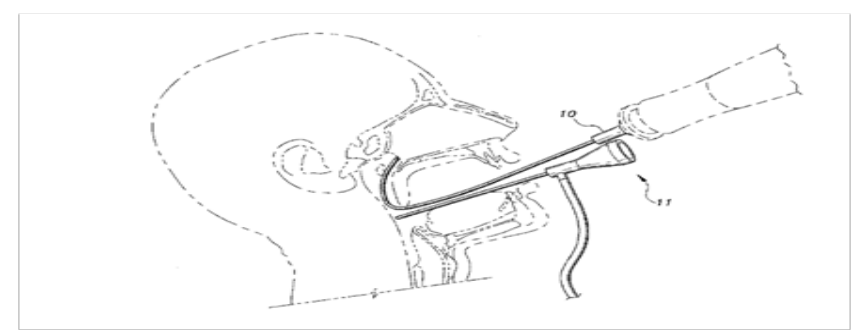

Figure 1 Diagrammatic perspective of the transoral approach for repair of choanal atresia.

\section{Advantages of invention}

i. Direct access to the area of narrowing

ii. Better and wider space to work in young children instead of working through narrow nasal passages

iii. Reduces intranasal trauma and subsequent narrowing

iv. Allows better resection of pathology (atretic plate and widened vomer)

v. Reduces the need for multiple revision surgeries

\section{Patent registry}

A patent titled "transoral repair of choanal atresia" was issued on Nov 29th 2016 from the United States patent office (US 9,504,454 B2). We are currently working on developing the instruments that will help us carry out the new surgical approach that we are proposing.

\section{Acknowledgment}

None.

\section{Conflict of interest}

None.

\section{References}

1. Gallagher TQ, Hartnick CJ. Endoscopic choanal atresia repair. $A d v$ Otorhinolaryngol. 2012;73:127-131.

2. Gulsen S. Treatment of Congenital Choanal Atresia via Transnasal Endoscopic Method. J Craniofac Surg. 2017;28(2):338-342.

3. Kwong KM. Current updates on choanal atresia. Front Pediatr. 2015;3:52.

4. Saraniti C, Santangelo M, Salvago P. Surgical treatment of choanal atresia with transnasal endoscopic approach with stentless single side-hinged flap technique: 5 year retrospective analysis. Braz J Otorhinolaryngol. 2017;83(2):183-189.

5. Kinis V, Ozbay M, Akdag M, et al. Patients with congenital choanal atresia treated by transnasal endoscopic surgery. J Craniofac Surg. 2014;25(3): 892-897.

6. Karligkiotis A. An Italian multicentre experience in endoscopic endonasal treatment of congenital choanal atresia: Proposal for a novel classification system of surgical outcomes. J Craniomaxillofac Surg. 2017;45(6):1018 1025 .

7. Brihaye P, Delpierre I, De Villé A, et al. Comprehensive management of congenital choanal atresia. Int J Pediatr Otorhinolaryngol. 2017;98:9-18.

8. Emel Çadallı Tatar, Bülent Öcale, Esra Doğan, et al. Stentless endoscopic repair of congenital choanal atresia: is it enough for maintaining choanal patency? European Archives of Oto-Rhino-Laryngology. 2017;274(10):3673-3678. 